\title{
Desafíos de la educación superior en el Perú durante la pandemia por la covid-19
}

\section{Challenges for higher education in Peru during the COVID-19 pandemic}

\section{Patty Vilela ${ }^{1}$}

Pontificia Universidad Católica del Perú

patty.vilelaa@pucp.pe

ORCID: https://orcid.org/0000-0002-8324-4478

Javier Sánchez ${ }^{2}$

Pontificia Universidad Católica del Perú

javier.sanchezc@pucp.edu.pe

ORCID: https://orcid.org/0000-0002-8551-761X

Cecilia Chau ${ }^{3}$

Pontificia Universidad Católica del Perú

cchau@pucp.edu.pe

ORCID: https://orcid.org/0000-0002-2631-0301

Citar como: Vilela, P., Sánchez, J. y Chau, C. (2021). Desafíos de la educación superior en el Perú durante la pandemia por la covid-19. Desde el Sur, 13(2), e0016.

\begin{abstract}
RESUMEN
El presente ensayo describe y reflexiona sobre la experiencia de la educación superior en el contexto de la emergencia sanitaria generada por la covid-19 y declarada desde marzo de 2020 hasta la actualidad. El cierre de las instalaciones de las universidades ha generado grandes retos para los estudiantes y docentes de todo el país. Frente a este contexto, se implementó la educación virtual con la finalidad de reducir los contagios y también garantizar la continuidad de los estudios de los jóvenes. La educación
\end{abstract}

\footnotetext{
1 Magíster en Salud Pública por la Universidad Pública de Navarra (España) y docente a tiempo parcial del Departamento de Psicología de la Pontificia Universidad Católica del Perú (PUCP). Es miembro del Grupo de Investigación Psicología y Salud: Entornos Saludables de la PUCP.

2 Magíster en Investigación en Ciencias Sociosanitarias por la Universidad de Alcalá (España) y docente a tiempo parcial del Departamento de Psicología de la PUCP. Es miembro del Grupo de Investigación Psicología y Salud: Entornos Saludables de la PUCP.

3 Doctora por la Universidad de Leuven (Bélgica) y docente principal a tiempo completo del Departamento de Psicología de la PUCP. Es coordinadora del Grupo de Investigación en Psicología de las Adicciones y Comportamientos de Riesgo (GI-ACR-PUCP). Es miembro del Grupo de Investigación Psicología y Salud: Entornos Saludables de la PUCP.
} 
virtual propició cambios, los cuales, en su mayoría, fueron forzados y poco planificados; no obstante, también han representado una oportunidad para la implementación de plataformas y tecnologías para el aprendizaje. A partir de la revisión y el análisis de la literatura científica, en este documento se discuten las implicancias para estudiantes y docentes en este nuevo escenario de la educación superior en el Perú.

\section{PALABRAS CLAVE}

Educación superior, pandemia, covid-19, docentes, estudiantes

\section{ABSTRACT}

This essay describes and reflects on the experience of higher education in the context of the public health emergency resulting from COVID-19 and declared from March 2020 to the present. The closure of university settings has created considerable challenges for students and teachers nationwide. In this context, virtual education was implemented, in order to reduce infections and also to ensure the continuity of studies for young people. Virtual education brought about changes, most of which were forced and unplanned; however, they have also constituted an opportunity for the implementation of learning platforms and technologies. Based on a review and analysis of the scientific literature, this paper discusses the implications for students and teachers of this new virtual education scenario in Peru.

\section{KEYWORDS}

Higher education, pandemic, COVID-19, teaching staff, students

\section{Introducción}

En el Perú se declaró el estado de emergencia nacional el 11 de marzo de 2020, con el Decreto Supremo 044-2020-PCM (Decreto Supremo 044-2020-PCM, 2019), que dispuso el aislamiento social obligatorio (cuarentena), el cierre temporal de las actividades no esenciales, entre otras medidas. Estas disposiciones se plantearon con la finalidad de evitar la propagación de la covid-19 en el país. En este escenario, las instituciones privadas y públicas de educación básica, alternativa y 
superior dispusieron a suspender sus actividades presenciales por un periodo de 15 días (Ministerio de Educación, 2020; Resolución Ministerial 085-2020-MINEDU). Progresivamente, esta medida se fue ampliando, conforme la emergencia sanitaria se fue agravando.

Esta disposición planteó que la enseñanza sería no presencial, lo cual implicó el desarrollo de estrategias para la implementación de la educación virtual, con la finalidad de garantizar la calidad y continuidad de los estudios de los jóvenes (Canaza-Choque, 2020). En este desafiante contexto, los docentes han tenido que rediseñar sus contenidos y aprender a utilizar nuevas plataformas virtuales (Portillo et al., 2020). De la misma manera, para los estudiantes este paso a la virtualidad ha implicado cambios y desafíos a varios niveles. Esta transición ha generado diversas dificultades y posturas contrapuestas sobre las mejores decisiones que se deben tomar a nivel metodológico y administrativo para los estudiantes universitarios (Fernández et al., 2020; Jiménez y Ruiz, 2021).

Las circunstancias en las que la educación virtual se desarrolló variaron de acuerdo con los recursos de las universidades, los docentes y los estudiantes. En este escenario, la desigualdad, presente desde antes de la pandemia, se hizo aún más visible y problemática (Acosta, 2020; CanezaChoque, 2020). Una arista de la desigualdad que se ha hecho notar en este contexto de pandemia es la brecha digital. En nuestro país, según el Instituto Nacional de Estadística e Información (INEI), el acceso a las tecnologías de la información y la comunicación (TIC) varía según el nivel de educación del jefe o la jefa de familia, es decir, las personas con mayor nivel de educación tienen una mayor cobertura de TIC. Asimismo, el 66\% de los hogares de Lima Metropolitana cuenta con acceso a Internet, en contraste con el 9,9\% de hogares procedentes del área rural del país (INEI, 2021).

Si bien la pandemia ha forzado la aceleración de la cobertura de este servicio a nivel nacional (INEI, 2021), la desigualdad en su acceso se mantiene según el lugar de procedencia y tipo de universidad en población universitaria. En una investigación realizada por HuancaArohuanca et al. (2020), en una muestra de 260 estudiantes universitarios peruanos, se encontró que los estudiantes que residen en Lima y cursan sus estudios en universidades particulares tienen mayores oportunidades para responder a las demandas de la educación virtual, en comparación con los estudiantes de provincia y de universidades estatales. Este contexto pone en evidencia que existe un grupo de estudiantes con limitados o escasos recursos y, por otro lado, se encuentran a los estudiantes con mejores condiciones y facilidades para adaptarse a la enseñanza virtual (Portillo et al., 2020). 
Estos datos revelan que la desigualdad en el acceso a las TIC representa una barrera importante para que jóvenes de zonas menos favorecidas puedan continuar sus estudios, lo que obliga a que, en muchos casos, decidan interrumpir su formación universitaria. Asimismo, si bien el uso de las TIC ha cobrado mayor relevancia en la pandemia, es importante considerar que estas condiciones desiguales afectan la posibilidad de continuar los estudios de los jóvenes tanto en el escenario presencial como el virtual (Acosta, 2020).

\section{Desafíos de los estudiantes en la pandemia}

El cambio de la educación presencial a la educación virtual en la pandemia ha forzado la reconfiguración de los roles de los estudiantes y docentes en el aula. En este contexto, el docente asume un rol más orientador y facilitador, mientras que se espera que el alumno sea el "protagonista de su propio aprendizaje» (Rugeles et al., 2015; Huang et al., 2020). En ese sentido, el estudiante se debe caracterizar por la autodisciplina (control de la fuerza de voluntad) y el autoaprendizaje (capacidad de aprender de manera autónoma, activa y participativa) (Rugeles et al., 2015). No obstante, se debe tomar en consideración que las condiciones en las que se transitó de una educación presencial a una virtual de emergencia fueron, en cierta medida, forzadas y apresuradas por la pandemia, lo cual generó que el cambio esté cargado de incertidumbre y limitada planificación.

En efecto, el paso de la presencialidad a la virtualidad no fue valorado positivamente por los estudiantes (Azhari y Kurniawati, 2021; Lovón et al., 2020; Tejedor et al., 2020). Las condiciones y cambios generaron este descontento en la población estudiantil. En ese sentido, la educación virtual demandaba más tiempo de dedicación a los estudiantes que la educación presencial (Rosario-Rodríguez et al., 2020). Asimismo, la insuficiente preparación por parte de los docentes generó una sobrecarga en el uso de herramientas como foros, tareas o lecturas, y ello ocasionó una mayor percepción de estrés en los estudiantes. Lovón y Cisneros (2020) exploraron estas dificultades en un grupo de 74 estudiantes de una universidad privada de Lima Metropolitana y encontraron que el 93,2\% de los estudiantes percibió que la carga académica había aumentado en comparación con el último semestre presencial (2019-1). En la misma línea, la mayoría de los estudiantes reportó percibir estrés y ansiedad. A nivel cualitativo, los investigadores destacan que los estudiantes reconocen un menor acompañamiento por parte de los docentes en la modalidad virtual; sin embargo, también son conscientes de que esta modalidad de enseñanza ha implicado un mayor esfuerzo por parte sus docentes. 
En otro estudio realizado con 116 estudiantes de educación media y superior en el estado de Sonora (México) se encontró que los estudiantes han experimentado nostalgia por el modelo de enseñanza presencial, específicamente por la interacción con los docentes, el desarrollo de las clases y la convivencia con sus compañeros (Portillo et al., 2020). En la misma línea, Tejedor et al. (2020) realizaron un estudio con 300 jóvenes universitarios de España, Italia y Ecuador, y encontraron que, en los tres países, los estudiantes reportaban que la disminución de la interacción con sus docentes representa una de las principales pérdidas en este contexto de educación virtual. De esta manera, se encuentra que el contacto físico con compañeros y docentes es añorado por los estudiantes en un momento en el que se exige tomar distancia del otro para protegerse.

Estos cambios en las relaciones sociales y las modificaciones de las condiciones del espacio educativo, sumados a la incertidumbre, el temor, el estrés y la ansiedad asociados a la pandemia, generan un impacto en la salud mental de la población. Con la finalidad de conocer el efecto de la pandemia en la población estudiantil en el Perú, Vivanco-Vidal et al. (2020) realizaron un estudio con 356 jóvenes universitarios de universidades públicas y privadas de las ciudades de Trujillo y Chimbote. Los resultados reafirman el efecto negativo de la pandemia en la salud mental en este grupo. Asimismo, los autores advierten que algunos grupos presentan un mayor grado de vulnerabilidad. Específicamente, las mujeres, los estudiantes que tienen amigos o familiares muy cercanos diagnosticados con covid-19, los jóvenes que no tienen trabajo y, por último, quienes pasan un tiempo aproximado de siete horas inmersos en noticias relacionadas con la covid-19 presentaron mayores niveles de ansiedad.

Las circunstancias actuales tienen un impacto en la salud mental de los jóvenes, como lo revela el estudio anterior. No obstante, aún es temprano para valorar con amplitud las dimensiones de sus consecuencias, porque el panorama es aún incierto y, al parecer, todavía sobrevendrán más retos. No obstante, a grandes luces se vislumbran cambios en la manera de relacionarnos y de aprender. Los estudios realizados en el último año, a nivel nacional e internacional, alertan sobre estos cambios y malestares que están experimentando los jóvenes estudiantes (Huanca-Arohuanca et al., 2020; Lovón et al., 2020; Portillo et al., 2020; Rosario-Rodríguez et al., 2020; Tejedor et al., 2020; Vivanco-Vidal et al., 2020). Para responder a esta demanda, se requerirá el fortalecimiento de los servicios de acompañamiento estudiantil, monitoreo psicopedagógico y los servicios médicos que disponen las universidades.

Por otro lado, a nivel mundial se han considerado ciertos criterios para priorizar a las poblaciones en mayor riesgo (Ministerio de Salud, 2020). 
Por ello, el plan de vacunación nacional contempla que los jóvenes serán el último grupo en ser inmunizado. Además, debido a la limitada oferta de vacunas a nivel mundial, todo parecer indicar que esta población será la última en retomar sus actividades en lo que será la «nueva normalidad» tras la covid-19.

En síntesis, el cambio a la educación virtual ha evidenciado la desigualdad y las dificultades que han presentado los estudiantes para adaptarse a este nuevo entorno de aprendizaje. Asimismo, se reconoce que este grupo no ha sido el único que ha debido movilizar sus recursos para adaptarse a los cambios imprevistos. Los docentes también han tenido que rediseñar y reajustar la metodología de enseñanza y evaluación en un escenario incierto y a un paso acelerado, como así lo demanda la pandemia.

\section{Desafíos de los docentes en la pandemia}

En este apartado, se brinda una aproximación de la vivencia del profesorado en este contexto. Este grupo parece estar recibiendo menor atención en las investigaciones, a pesar de que están expuestos a situaciones altamente complejas e inciertas, como la renovación de sus contratos, la reducción del personal docente (Instituto Internacional para la Educación Superior en América Latina y el Caribe, 2020) y otros reajustes pedagógicos ocasionados por la virtualidad. Para comprender estos cambios es importante tomar en cuenta que las modificaciones en el proceso de enseñanza de la presencialidad a la virtualidad podrían ser más complejas en ciertas áreas del conocimiento que requieren de la asistencia a las aulas de los campus universitarios, porque necesitan ciertas herramientas (laboratorios o equipos, por ejemplo).

La evidencia existente de distintos países coincide en destacar que la falta de una infraestructura adecuada para la virtualidad, la falta de experiencia, la alta exigencia docente, la brecha de acceso a la información, la falta de adecuación de los ambientes de estudio o trabajo (en muchos casos improvisados) y la incertidumbre constante representan retos muy complejos y que son importantes de reconocer y profundizar (Marshall et al., 2020; Pokhrel y Chhetri, 2021; Pressley, 2021; Román, 2020). Otro aspecto, quizás menos mencionado, es el económico. El recorte de presupuesto producto de la deserción estudiantil podría implicar que las universidades no puedan atender todas las necesidades del profesorado (Figallo et al., 2020). El impacto negativo en las condiciones laborales de los docentes ha generado un contexto hostil y de incertidumbre laboral, el cual ha podido impactar en su desempeño en clase (Quezada et al., 2021). 
Cabe señalar que el cambio forzado e inesperado de la virtualidad ha generado que los docentes aprendan e incorporen las TIC a los procesos de aprendizaje en corto tiempo (Bao, 2020; Canaza-Choque, 2020; Valdivia, 2020). Además, a pesar de las adversidades, los docentes han mantenido el desempeño y la calidad de enseñanza, al igual que en la presencialidad (Instituto Internacional para la Educación Superior en América Latina y el Caribe, 2020). No obstante, las condiciones no han sido favorables, dado que la mayoría de las universidades en el Perú no habían desarrollado la infraestructura digital que requiere la educación virtual, por lo que este aprendizaje se ha desarrollado sobre la marcha. A este panorama se le suma también la búsqueda del equilibrio entre la vida laboral y la familiar de los docentes y la implementación del teletrabajo en casa (Dos Santos et al., 2021).

En este contexto, se pueden rescatar aspectos positivos y recursos muy útiles en la virtualidad; no obstante, no se debe perder de vista que se trata de un contexto de emergencia. La revisión de Almazova et al. (2020) muestra que existen estudios que destacan las bondades de la enseñanza virtual, pero han sido elaborados en contextos anteriores a la pandemia. Por tanto, es natural que la vivencia de los docentes no se produzca como la literatura previa sugiere. Se debe considerar que pueden existir dificultades incluso cuando la transición a la virtualidad sea gradual (Marshall et al., 2020). Además, muchos de esos esfuerzos no siempre apuntan a la raíz del problema. Existen experiencias como las de Fernández et al. (2020) que muestran que las capacitaciones institucionales a docentes incluyen recomendaciones de forma (qué plataformas virtuales emplear) más que de fondo (cómo adaptar su contenido a la educación virtual).

Por último, es importante resaltar que, a pesar de las adversidades, esta etapa de cambios ha servido para el descubrimiento de un sinnúmero de herramientas digitales y TIC que, sin duda alguna, serán útiles también cuando se retomen las clases presenciales. La potenciación de la enseñanza tradicional en un aula física, a través de recursos digitales, permitirá facilitar el proceso de enseñanza en la educación superior. Asimismo, es importante destacar que, en el contexto de la pandemia, la labor docente resulta más compleja, ya que las brechas en el acceso a las TIC se han acentuado y no todos los estudiantes y docentes cuentan con las mismas oportunidades de conectividad, como se mencionó inicialmente.

\section{Conclusiones}

A pesar de las resistencias iniciales, la virtualización de la educación ha significado una oportunidad para implementar cambios importantes en 
este sector, que en otras circunstancias habrían sido difíciles de imaginar. Por ello, se ha considerado importante realizar la presente revisión, la cual ha permitido una aproximación a la experiencia de la transición a la educación virtual de docentes y estudiantes en el Perú.

Se puede concluir que, pese a las adversidades, esta etapa también ha generado oportunidades de aprendizaje y desarrollo para la comunidad universitaria. En efecto, los estudiantes destacan ganancias a nivel personal, como una mayor autodisciplina, una mejor gestión del tiempo, responsabilidad, resiliencia, autonomía y flexibilidad (Lovón et al., 2020; Portillo et al., 2020; Rosario-Rodríguez et al., 2020; Román, 2020; Tejedor et al., 2020). Por parte de los docentes, este paso a la educación virtual ha permitido el desarrollo de diversas competencias y herramientas digitales que representan un aprendizaje valioso.

Por último, si bien se destacan aspectos positivos de este periodo, también es importante tomar en cuenta el desgaste generado por los desafíos que implica la educación a distancia. Por ello, es necesario que se continúe investigando en el impacto de estos cambios para plantear propuestas de intervención que contribuyan con la prevención de problemas que puedan afectar la salud y el bienestar de los estudiantes y los docentes.

\section{Contribución de autoría}

Patty Vilela, Javier Sánchez y Cecilia Chau contribuyeron con la búsqueda de información y redacción del ensayo.

\section{Fuente de financiamiento}

Autofinanciado.

\section{Potenciales conflictos de interés}

Ninguno. 


\section{REFERENCIAS BIBLIOGRÁFICAS}

Acosta, A. (2020). La educación superior ante el Covid-19. Un nuevo reto y viejos resabios. http://www5.diputados.gob.mx/index.php/camara/ Centros-de-Estudio/CESOP/Tema-Covid-19/Reporte-CESOP.-Covid-19-LaHumanidad-a-Prueba.-Edicion-Especial.-Num.-132-mayo-2020

Almazova, N., Krylova, E., Rubtsova, A. y Odinokaya, M. (2020). Challenges and opportunities for Russian higher education amid covid-19: Teachers' perspective. Education Sciences, 10(12), 1-11. https://doi.org/10.3390/ educsci10120368

Azhari, T. y Kurniawati, T. (2021). Students' perception on online learning during the Covid-19 pandemic: A Casecstudy of Universitas Malikussaleh students. Proceedings of the International Conference on Social Science, Political Science, and Humanities (ICOSPOLHUM 2020). International Conference on Social Science, Political Science, and Humanities (ICoSPOLHUM 2020), Lhokseumawe, Indonesia. https://doi.org/10.2991/ assehr.k.210125.009

Bao, W. (2020). COVID-19 and online teaching in higher education: A case study of Peking University. Human Behavior and Emerging Technologies, 2(2), 113-115. https://doi.org/10.1002/hbe2.191

Canaza-Choque, F. A. (2020). Educación superior en la cuarentena global: disrupciones y transiciones. Revista Digital de Investigación en Docencia Universitaria, 14(2), e1315. https://doi.org/10.19083/10.19083/ ridu.2020.1315

Decreto Supremo 044-2020-PCM (2019). Decreto Supremo que declara Estado de Emergencia Nacional por las graves circunstancias que afectan la vida de la Nación a consecuencia del brote del COVID-19. https:// busquedas.elperuano.pe/normaslegales/decreto-supremo-que-declaraestado-de-emergencia-nacional-po-decreto-supremo-n-044-2020pcm-1864948-2/

Dos Santos, B. Scorsolini-Comin, F. y De Cassia, R. (2021). Ser docente en el contexto de la pandemia de COVID-19: reflexiones sobre la salud mental. Inex de Enfermería, 29(3). https://scielo.isciii.es/scielo.php?script=sci_artte $x t \& p i d=S 1132-12962020000200008$

Fernández, U., Gewerc Barujel, A. y Llamas Nistal, M. (2020). El profesorado universitario de Galicia y la enseñanza remota de emergencia: condiciones y contradicciones. Campus Virtuales: Revista Científica Iberoamericana de Tecnología Educativa, 9, 9-24.

Figallo, F., González, M. T. y Diestra, V. (2020). Perú: Educación superior en el contexto de la pandemia por el COVID-19. Revista de Educación Superior en América Latina, 8, 20-28. https://doi.org/10.14482/esal.8.378.85 
Huanca-Arohuanca, J., Supo-Condori, F., Sucari, R. y Supo, L. (2020). El problema social de la educación virtual universitaria en tiempos de pandemia, Perú. Revista Innovaciones Educativas, 22, 115-128. https://doi. org/10.22458/ie.v22iespecial.3218

Huang, R. H., Liu, D. J., Amelina, N., Yang, J. F., Zhuang, R. X., Chang, T. W. y Cheng, W. (2020). Guidance on active learning at home during educational disruption: Promoting student's self-regulation skills during COVID-19 outbreak. Beijing. https://iite.unesco.org/wp-content/uploads/2020/04/ Guidance-on-Active-Learning-at-Home-in-COVID-19-Outbreak.pdf

Instituto Internacional para la Educación Superior en América Latina y el Caribe. (2020). COVID-19 y educación superior: De los efectos inmediatos al día después. Análisis de impactos, respuestas políticas y recomendaciones. Unesco, 44. http://www.iesalc.unesco.org/wp-content/ uploads/2020/04/COVID-19-060420-ES-2.pdf

Instituto Nacional de Estadística e Informática (marzo, 2021). Informe técnico. Estadísticas de las tecnologías y comunicación en los hogares. Trimestre octubre-noviembre-diciembre 2020. https://www.inei.gob. $\mathrm{pe/media/MenuRecursivo/boletines/01-informe-tecnico-tic-iv-}$ trimestre-2020.pdf

Jiménez, Y. y Ruiz, M. A. (2021). Reflexiones sobre los desafíos que enfrenta la educación superior en tiempos de COVID-19. Economía y Desarrollo, 165(1). http://scielo.sld.cu/scielo.php?script=sci_arttext\&pid $=$ S0252-85842021000200003

Lovón, M. y Cisneros, S. (2020). Repercusiones de las clases virtuales en los estudiantes universitarios en el contexto de la cuarentena por COVID-19: El caso de la PUCP. Propósitos y Representaciones, 8 (SPE3), e588. http:// dx.doi.org/10.20511/pyr2020.v8nSPE3.588

Marshall, D. T., Shannon, D. M. y Love, S. M. (2020). How teachers experienced the COVID-19 transition to remote instruction. Phi Delta Kappan, 102(3), 46-50. https://doi.org/10.1177/0031721720970702

Ministerio de Educación. (2020). Orientaciones para la continuidad del servicio educativo superior universitario, en el marco de la emergencia sanitaria por el COVID-19. Ministerio de Educación, 2-7. http://www. minedu.gob.pe/reforma-universitaria/pdf/orientaciones-universidades. pdf

Ministerio de Salud. (2020). Nueva estrategia de vacunación contra la COVID-19 con enfoque territorial. https://www.gob.pe/11796-plannacional-de-vacunacion-contra-la-covid-19

Pokhrel, S. y Chhetri, R. (2021). A literature review on impact of COVID-19 pandemic on teaching and learning. Higher Education for the Future, 8(1), 133-141. https://doi.org/10.1177/2347631120983481 
Portillo, S., Castellanos, L., Reynoso, O. y Gavotto, O. (2020). Enseñanza remota de emergencia ante la pandemia Covid-19 en Educación Media Superior y Educación Superior. Propósitos y Representaciones, 8 (SPE3), e589. DOI: http://dx.doi.org/10.20511/pyr2020.v8nSPE3.589

Pressley, T. (2021). Factors contributing to teacher burnout during COVID-19. Educational Researcher, 84-86. https://doi. org/10.3102/0013189X211004138

Quezada, M. del P., Castro Arellano, M. del P., Dios Castillo, C. A. y Quezada Castro, G. A. (2021). Condiciones laborales en la educación universitaria peruana: Virtualización ante la pandemia COVID-19. Revista Venezolana de Gerencia, 26(93), 110-123. https://doi.org/10.52080/rvg93.09

Resolución Ministerial 080-2020- MINEDU (2020). Norma Técnica Orientaciones para la prevención, atención ante el Coronavirus (COVID-19) en los Centros de Educación Técnico-Productiva e Institutos y Escuelas de Educación Superior. https://cdn.www.gob.pe/uploads/document/ file/565535/RVM_N_080-2020-MINEDU.PDF

Román, J. A. M. (2020). La educación superior en tiempos de pandemia: una visión desde dentro del proceso formativo. Revista Latinoamericana de Estudios Educativos, 5. https://doi.org/10.48102/rlee.2020.50.ESPECIAL.95

Rosario-Rodríguez, A., González-Rivera, J. A., Cruz-Santos, A. y RodríguezRíos, L. (2020). Demandas tecnológicas, académicas y psicológicas en estudiantes universitarios durante la pandemia por COVID-19. Revista Caribeña de Psicología, 4(2), 176-185. https://doi.org/10.37226/rcp. v4i2.4915

Rugeles, P., Mora, B.y Metaute,P.(2015).El rol del estudianteen los ambientes educativos mediados por las TIC. Revista Lasallista de Investigación, 12(2), 132-138. https://www.redalyc.org/pdf/695/69542291025.pdf

Tejedor, S., Cervi, L., Tusa, F. y Parola, A. (2020). Educación en tiempos de pandemia: reflexiones de alumnos y profesores sobre la enseñanza virtual universitaria en España, Italia y Ecuador. Revista Latina de Comunicación Social, 78, 1-21. https://www.doi.org/10.4185/RLCS-2020-1466

Valdivia, P. (2020). Educación superior: Pandemia COVID-19. Revista Digital de Investigación en Docencia Universitaria, 14(2). https://doi.org/10.19083/ ridu. 2020.1388

Vivanco-Vidal, A., Saroli-Araníbar, D., Caycho-Rodríguez, T., Carbajal-León, C. y Noé-Grijalva, M. (2020). Ansiedad por Covid-19 y salud mental en estudiantes universitarios. Revista de Investigación en Psicología, 23(2), 197-216. http://dx.doi.org/10.15381/rinvp.v23i2.19241 